\title{
Malignant Primitive Cellular Infiltrate
}

National Cancer Institute

\section{Source}

National Cancer Institute. Malignant Primitive Cellular Infiltrate. NCI Thesaurus. Code C156460.

A morphologic finding indicating the presence of a malignant primitive cellular infiltrate in a tissue sample. 